\title{
Fragmentation functions of charged hadrons
}

\section{Emanuele R. Nocera*}

Rudolf Peierls Centre for Theoretical Physics, University of Oxford,

1 Keble Road, Oxford OX1 3NP, United Kingdom

E-mail: emanuele.nocera@physics.ox.ac.uk

I present a preliminary determination of the Fragmentation Functions (FFs) of unidentified charged hadrons at next-to-leading order in quantum chromodynamics. The analysis is based on hadron production cross section data in single-inclusive electron-positron annihilation. It extends a recent determination of the FFs of identified charged pions, charged kaons and protons/antiprotons performed by the NNPDF Collaboration. I illustrate the quality of the FFs determined in this analysis and show how they describe the charged hadron spectra measured in proton-(anti)proton collisions.

XXV International Workshop on Deep-Inelastic Scattering and Related Subjects 3-7 April 2017

University of Birmingham, UK

\footnotetext{
*Speaker.
} 
In a recent paper [1], the NNPDF Collaboration presented NNFF1.0, a determination of the Fragmentation Functions (FFs) of charged pions, charged kaons and protons/antiprotons. The analysis included a comprehensive set of cross section measurements for single-inclusive hadron production in electron-positron annihilation (SIA). It was performed within the NNPDF methodology - a fitting framework designed to provide a statistically sound representation of FF uncertainties with a minimal procedural bias - at leading, next-to-leading and next-to-next-to-leading order (LO, NLO and NNLO) in perturbative Quantum Chromodynamics (QCD). The systematic inclusion of higher-order QCD corrections allowed the ensuing NNFF1.0 FF sets to describe the data very well, including those points at rather small hadron momentum fractions $z$. Together with existing sets of unpolarised [2] and polarised [3] Parton Distribution Functions (PDFs), FFs and PDFs are available from a common, mutually consistent framework for the first time.

A number of hard-scattering processes, probing nucleon momentum, spin, flavour, spatial distributions and the dynamics of nuclear matter [4] require the knowledge of FFs. In some of them, charged hadrons are inclusively measured in the final state, without identifying the hadronic species. In this case, although charged pions, kaons and protons/antiprotons dominate the hadron yields, FFs accounting also for the residual hadron fraction should be used.

In this contribution, I extend the NNFF1.0 analysis to a preliminary determination of the FFs of unidentified charged hadrons, though only at NLO. As in NNFF1.0, I restrict to SIA, and I consider flavour-untagged (inclusive) and tagged measurements performed by experiments at CERN (ALEPH [7], DELPHI [8, 9] and OPAL [10, 11]), DESY (TASSO [5]) and SLAC (TPC [6] and SLD [12]). The included data set is summarised in Tab. 1, where I specify, in the first five columns, the name of the experiment, the corresponding publication reference, the measured observable, the center-of-mass energy $\sqrt{s}$, and the number of data points included in the fit after (before) applying kinematic cuts. On top of total inclusive and tagged cross section measurements, longitudinal inclusive and tagged cross section data are also available for unidentified charged hadrons, in contrast to identified charged pions, charged kaons and charged protons/antiprotons. They are proportional respectively to the structure functions $F_{2}^{h^{+}+h^{-}}$and $F_{L}^{h^{+}+h^{-}}$. The latter is more sensitive to the gluon FF than the former, because it includes a non-vanishing $\mathscr{O}\left(\alpha_{s}\right)$ contribution, with $\alpha_{s}$ the QCD strong coupling, already at LO. This helps constraining the gluon FF, especially since the lack of precise data over a wide range of energies makes it only roughly determined by evolution.

The theoretical and methodological details of this analysis, including the description of the experimental observables in terms of the FFs, their parametrisation, and the fitting procedure, are exactly the same as in the NNFF1.0 analysis. Kinematic cuts are choosen consistently. Data points below $z_{\min }$, with $z_{\min }=0.02$ for experiments at $\sqrt{s}=M_{Z}, z_{\min }=0.075$ for all other experiments, and $z_{\max }=0.9$ are not included in this fit, as in NNFF1.0.

In the last column of Tab. 1 , I report the $\chi^{2}$ per number of data points, $\chi^{2} / N_{\text {dat }}$, for both the individual and total data sets. The data/theory ratio is diplayed for each data set in Fig. 1. Bands correspond to one- $\sigma$ FF uncertainties, and shaded areas indicate the regions excluded by kinematic cuts. As is apparent from both Tab. 1 and Fig. 1, the overall fit quality, measured by the total $\chi^{2} / N_{\text {dat }}=0.83$, is very good. All data sets are very well described, with the exception of the OPAL inclusive data set. The large value of the $\chi^{2}$ per number of data points, $\chi^{2} / N_{\text {dat }}=2.41$, is determined by a handful of rather precise data points at large values of $z$. A similar behaviour was already observed in the NNFF1.0 analysis for the individual hadron species, where a certain 


\begin{tabular}{|c|c|c|c|c|c|}
\hline Experiment & Reference & Observable & $\sqrt{s}[\mathrm{GeV}]$ & $N_{\text {dat }}$ & $\chi^{2} / N_{\text {dat }}$ \\
\hline TASSO14 & [5] & $\frac{1}{\sigma_{\mathrm{tot}}} \frac{d \sigma^{h^{ \pm}}}{d z}$ & 14.00 & $15(20)$ & 1.23 \\
\hline TASSO 22 & [5] & $\frac{1}{\sigma_{\mathrm{tot}}} \frac{d \sigma^{h^{ \pm}}}{d z}$ & 22.00 & $15(20)$ & 0.51 \\
\hline ТPC & [6] & $\frac{1}{\sigma_{\text {tot }}} \frac{d \sigma^{h^{ \pm}}}{d z}$ & 29.00 & $21(34)$ & 1.65 \\
\hline TASSO35 & [5] & $\frac{1}{\sigma_{\text {tot }}} \frac{d \sigma^{h^{ \pm}}}{d z}$ & 35.00 & $15(20)$ & 1.14 \\
\hline TASSO44 & [5] & $\frac{1}{\sigma_{\mathrm{tot}}} \frac{d \sigma^{h^{ \pm}}}{d z}$ & 44.00 & $15(20)$ & 0.68 \\
\hline \multirow[t]{2}{*}{ ALEPH } & [7] & $\frac{1}{\sigma_{\text {tot }}} \frac{d \sigma^{h^{ \pm}}}{d z}$ & 91.20 & $32(35)$ & 1.04 \\
\hline & [7] & $\frac{1}{\sigma_{\mathrm{tot}}} \frac{d \sigma_{L}^{h^{ \pm}}}{d z}$ & 91.20 & $19(21)$ & 0.36 \\
\hline \multirow[t]{5}{*}{ DELPHI } & [8] & $\frac{1}{\sigma_{\mathrm{tot}}} \frac{d \sigma^{h^{ \pm}}}{d p_{h}}$ & 91.20 & $21(27)$ & 0.65 \\
\hline & [8] & $\left.\frac{1}{\sigma_{\text {tot }}} \frac{d \sigma^{h^{ \pm}}}{d p_{h}}\right|_{u d s}$ & 91.20 & 21 (27) & 0.17 \\
\hline & [8] & $\left.\frac{1}{\sigma_{\mathrm{tot}}} \frac{d \sigma^{h^{ \pm}}}{d p_{h}}\right|_{b}$ & 91.20 & $21(27)$ & 0.82 \\
\hline & [9] & $\frac{1}{\sigma_{\mathrm{tot}}} \frac{d \sigma_{L}^{h^{ \pm}}}{d z}$ & 91.20 & $20(22)$ & 0.72 \\
\hline & [9] & $\left.\frac{1}{\sigma_{\mathrm{tot}}} \frac{d \sigma_{L}^{h^{ \pm}}}{d z}\right|_{b}$ & 91.20 & $20(22)$ & 0.44 \\
\hline \multirow[t]{5}{*}{ OPAL } & [10] & $\frac{1}{\sigma_{\mathrm{tot}}} \frac{d \sigma^{h^{ \pm}}}{d z}$ & 91.20 & $20(22)$ & 2.41 \\
\hline & [10] & $\left.\frac{1}{\sigma_{\mathrm{tot}}} \frac{d \sigma^{h^{ \pm}}}{d z}\right|_{u d s}$ & 91.20 & $20(22)$ & 0.90 \\
\hline & [10] & $\frac{1}{\sigma_{\mathrm{tot}}} \frac{d \sigma^{h^{ \pm}}}{d z}$ & 91.20 & $20(22)$ & 0.61 \\
\hline & [10] & $\frac{1}{\sigma_{\mathrm{tot}}} \frac{d \sigma^{h^{ \pm}}}{d z}$ & 91.20 & $20(22)$ & 0.21 \\
\hline & [11] & $\frac{1}{\sigma_{\text {tot }}} \frac{d \sigma_{L}^{h^{ \pm}}}{d z}$ & 91.20 & $20(22)$ & 0.31 \\
\hline \multirow[t]{4}{*}{ SLD } & [12] & $\frac{1}{\sigma_{\mathrm{tot}}} \frac{d \sigma^{h^{ \pm}}}{d p_{h}}$ & 91.28 & $34(40)$ & 0.75 \\
\hline & [12] & $\left.\frac{1}{\sigma_{\mathrm{tot}}} \frac{d \sigma^{h^{ \pm}}}{d z}\right|_{u d s}$ & 91.28 & $34(40)$ & 1.03 \\
\hline & [12] & $\frac{1}{\sigma_{\mathrm{tot}}} \frac{d \sigma^{h^{ \pm}}}{d z}$ & 91.28 & $34(40)$ & 0.62 \\
\hline & [12] & $\left.\frac{1}{\sigma_{\mathrm{tot}}} \frac{d \sigma^{h^{ \pm}}}{d z}\right|_{b}$ & 91.28 & $34(40)$ & 0.97 \\
\hline Total dataset & & & & 471 (527) & 0.83 \\
\hline
\end{tabular}

Table 1: The data set included in this analysis of FFs for unidentified charged hadrons. For each experiment, I indicate the publication reference, the measured observable, the center-of-mass energy $\sqrt{s}$, the number of data points included after (before) kinematic cuts, and the $\chi^{2}$ per number of data points, $\chi^{2} / N_{\text {dat }}$.

amount of tension between the OPAL data and the other data sets at $\sqrt{s}=M_{Z}$ was found. The data points below $z_{\min }$ are reasonably described by the extrapolated FFs for all data sets at $\sqrt{s}=M_{Z}$.

The FFs for the singlet, $D_{\Sigma}^{h^{ \pm}}=\sum_{q}^{n_{f}}=D_{q}^{h^{ \pm}}+D_{\bar{q}}^{h^{ \pm}}$(with $n_{f}$ the number of active flavours), gluon, $D_{g}$, and total charm and bottom, $D_{q^{+}}^{h^{ \pm}}=D_{q}^{h^{ \pm}}+D_{\bar{q}}^{h^{ \pm}}$(with $q=c, b$ ) are displayed in Fig. 2 at 

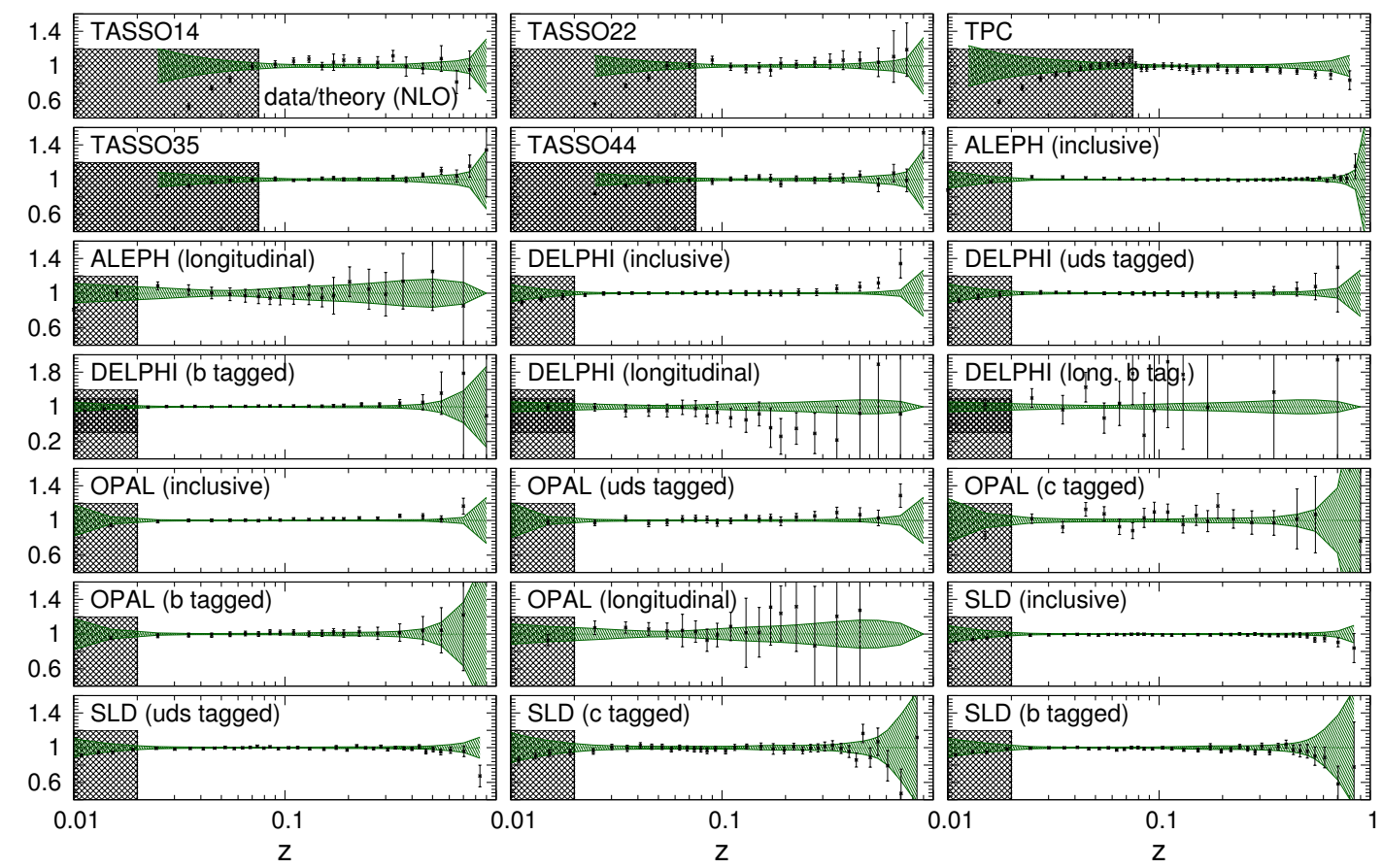

Figure 1: The data/theory ratio for the unidentified light charged hadron data included in this analysis. Theoretical predicitons are computed at NLO with the corresponding best-fit FFs. Bands correspond to one- $\sigma$ FF uncertainties. Shaded areas indicate the regions excluded by kinematic cuts.

$Q^{2}=M_{Z}^{2}$ as a function of $z$. The results from this analysis (denoted as NNPDF) include the one- $\sigma$ uncertainty bands. They are compared to the central value from the DSS analysis [13], to which they are also normalised in each panel below the corresponding FFs. Uncertainty bands on the DSS results are not shown because they are not available to the author.

As is apparent from Fig. 2, the most relevant discrepancy between this analysis and DSS is in the gluon FF, which is harder in the former than in the latter at small-to-medium values of $z$, $0.03 \lesssim z \lesssim 0.3$. It is instead softer at large values of $z, 0.3 \lesssim z \lesssim 0.9$, although compatible with the DSS central value within uncertainties. A similar behaviour was observed for the gluon FF of charged pions in NNFF1.0 [1]. At small- $z$, it was recognised to be a consequence of the less conservative small- $z$ kinematic cut adopted in [1] with respect to [13]; at large- $z$ of the more flexible NNPDF parametrisation. Furthermore, this determination, despite being based on a wider set of SIA data than [13], does not include proton-(anti)proton collider data. This data is directly sensitive to the gluon FF, it was taken into account in [13], and may also account for the differences observed. All other FFs in Fig. 2 are in reasonable agreement with the DSS central value, except at small $z$, again because of the different kinematic cut adopted in the two analyses.

As an example of a possible application, the FFs determined in this analysis are used to compute predictions for the differential cross section spectra as a function of the transverse momentum of the hadron, $p_{T}$. The results are obtained at NLO with the code presented in [14] and the PDF central value from the NNPDF3.1 set [2]. They are compared to measurements performed in proton-antiproton collisions by the $\mathrm{CDF}$ experiment at $\sqrt{s}=1.8$ and $1.96 \mathrm{TeV}$ [15], and in protonproton collisions by the CMS [16] and ALICE [17] experiments at $\sqrt{s}=0.9,2.76$ and $7 \mathrm{TeV}$. The 


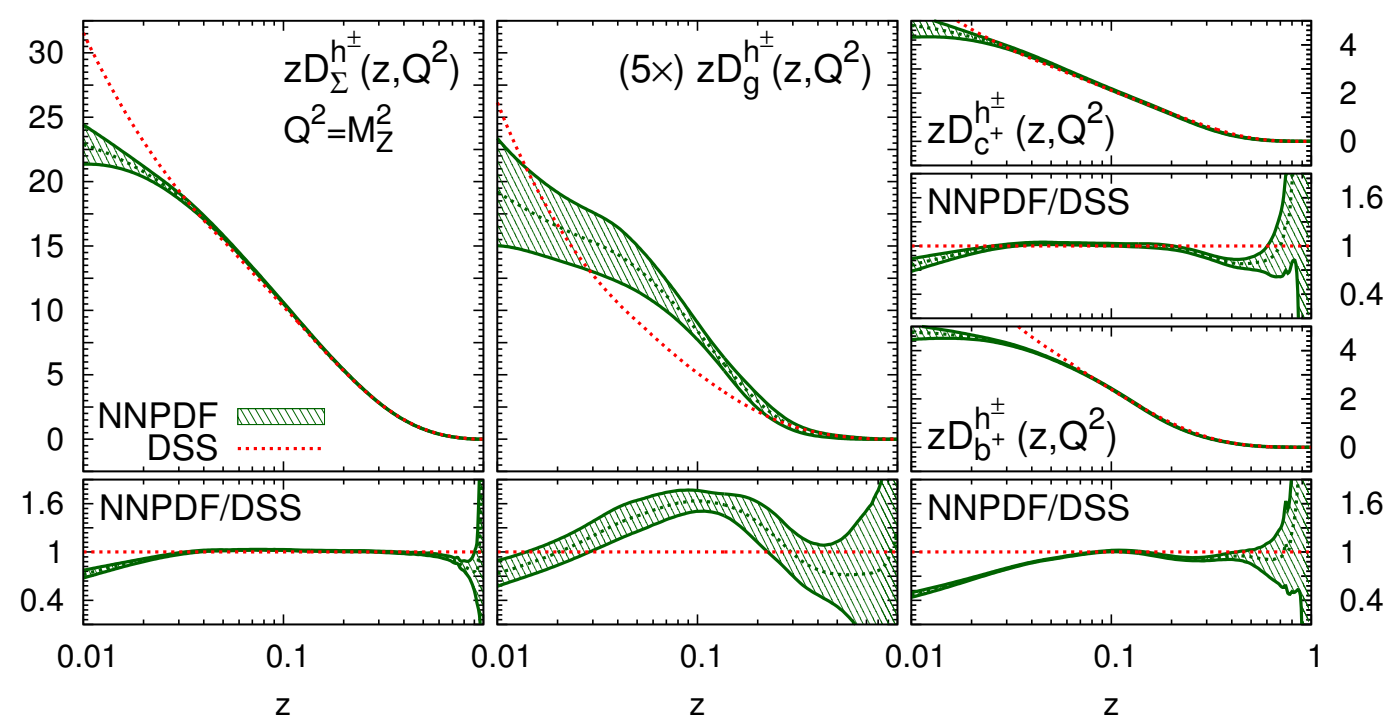

Figure 2: Comparison between the singlet, gluon, and total charm and bottom FFs for the unidentified charged hadrons obtained from this analysis (denoted as NNPDF) and the DSS set at $Q^{2}=M_{Z}^{2}$; NNPDF uncertainties correspond to one- $\sigma$ bands. The ratio to the DSS central value is also displayed below each FF.
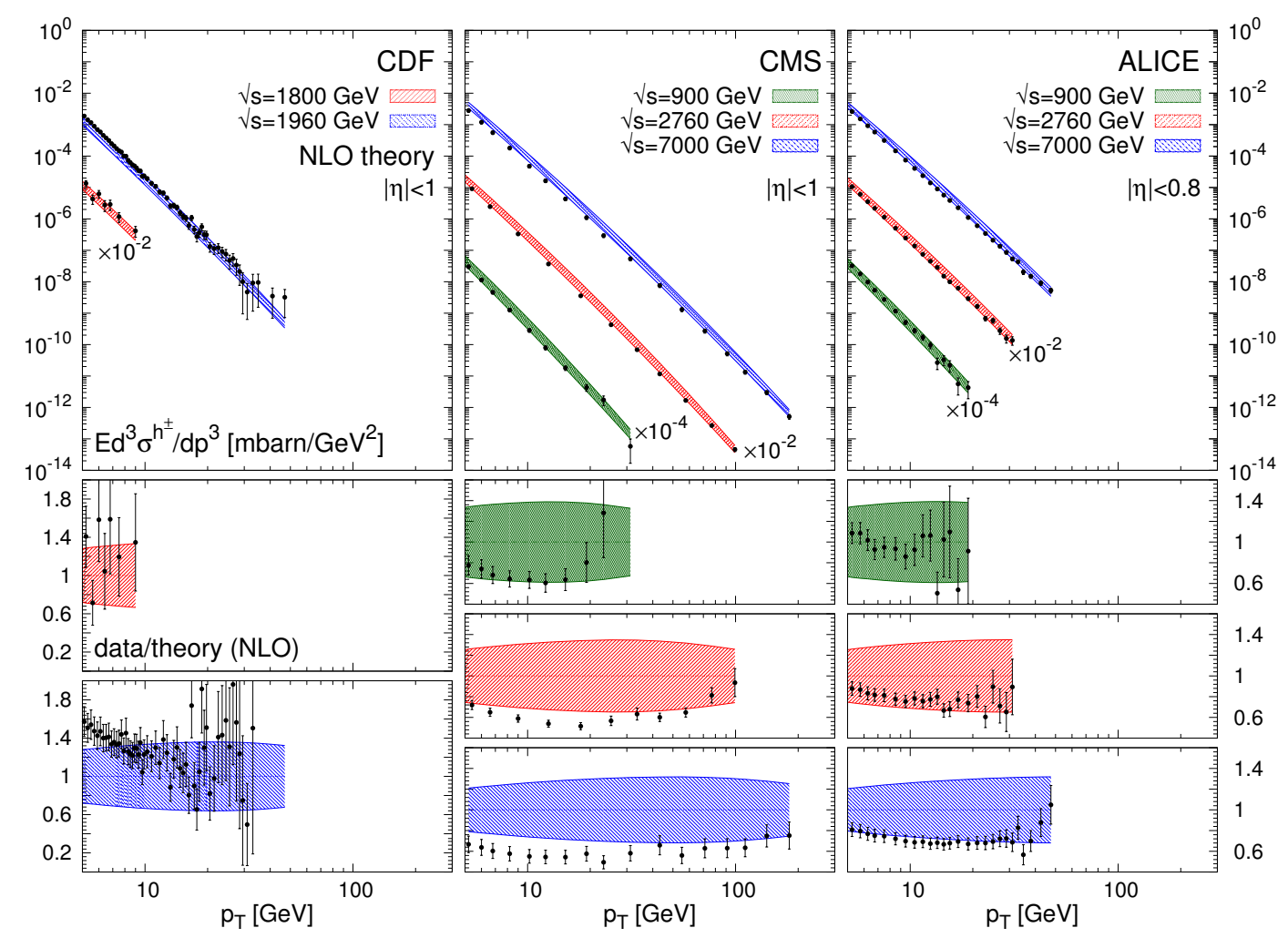

Figure 3: Differential cross section data for the inclusive charged hadron spectra measured by CDF, CMS and ALICE in proton-(anti)proton collisions at different center-of-mass energies $\sqrt{s}$ integrated over the rapidity $\eta$. The data is compared to the NLO predictions obtained with the FFs determiend in this analysis. The corresponding data/theory ratio is also shown. One- $\sigma$ bands include the FF uncertainty only. 
data/theory ratio is also shown. One- $\sigma$ uncertainty bands include the FF uncertainty only.

The data-theory agreement displayed in Fig. 3 is reasonable in almost all cases. Theoretical predictions tend to overshoot experimental data at the highest center-of-mass energies. However they remain mutually compatible within uncertainties, except in the case of the CMS experiment at $\sqrt{s}=2.76$ and $7 \mathrm{TeV}$. A similar behaviour was pointed out in [18], where all the FF sets available at that time were shown to grossly overshoot charged hadron spectra cross section data. In this analysis such an effect, though still partly present, is mitigated. The reason is related to the gluon FF, to which the observable in Fig. 3 is primarily sensitive. The gluon FF determined in this analysis is indeed rather different from the DSS one (and from the one in other similar analyses), see Fig. 2. Furthermore, it is affected by larger uncertainties, as explicitly shown for identified hadrons in [1]. Finally, the uncertainty of the data, especially from CMS and ALICE, is rather narrower than the uncertainty due to the FF. This suggests that the data will carry in a large amount of information on the gluon FF once included in a global FF fit [19].

I thank V. Bertone and L. Rottoli for their collaboration in sorting out the data and predictions shown in Fig. 3. This work is supported by the UK STFC Rutherford Grant ST/M003787/1.

\section{References}

[1] V. Bertone et al. [NNPDF Collaboration], Eur. Phys. J. C 77 (2017) 516.

[2] R. D. Ball et al. [NNPDF Collaboration], arXiv:1706.00428 [hep-ph].

[3] E. R. Nocera et al. [NNPDF Collaboration], Nucl. Phys. B 887 (2014) 276.

[4] S. Albino et al., arXiv:0804.2021 [hep-ph].

[5] W. Braunschweig et al. [TASSO Collaboration], Z. Phys. C 47 (1990) 187.

[6] H. Aihara et al. [TPC/Two Gamma Collaboration], Phys. Rev. Lett. 61 (1988) 1263.

[7] D. Buskulic et al. [ALEPH Collaboration], Phys. Lett. B 357 (1995) 487 Erratum: [Phys. Lett. B 364 (1995) 247].

[8] P. Abreu et al. [DELPHI Collaboration], Eur. Phys. J. C 5 (1998) 585.

[9] P. Abreu et al. [DELPHI Collaboration], Eur. Phys. J. C 6 (1999) 19.

[10] K. Ackerstaff et al. [OPAL Collaboration], Eur. Phys. J. C 7 (1999) 369.

[11] R. Akers et al. [OPAL Collaboration], Z. Phys. C 68 (1995) 203.

[12] K. Abe et al. [SLD Collaboration], Phys. Rev. D 69 (2004) 072003.

[13] D. de Florian, R. Sassot and M. Stratmann, Phys. Rev. D 76 (2007) 074033.

[14] B. Jager, A. Schafer, M. Stratmann and W. Vogelsang, Phys. Rev. D 67 (2003) 054005.

[15] F. Abe et al. [CDF Collaboration], Phys. Rev. Lett. 61 (1988) 1819; T. Aaltonen et al. [CDF Collaboration], Phys. Rev. D 79 (2009) 112005 Erratum: [Phys. Rev. D 82 (2010) 119903].

[16] S. Chatrchyan et al. [CMS Collaboration], JHEP 1108 (2011) 086; Eur. Phys. J. C 72 (2012) 1945.

[17] B. B. Abelev et al. [ALICE Collaboration], Eur. Phys. J. C 73 (2013) 2662.

[18] D. d'Enterria, K. J. Eskola, I. Helenius and H. Paukkunen, Nucl. Phys. B 883 (2014) 615.

[19] V. Bertone, N. P. Hartland, E. R. Nocera and L. Rottoli, in preparation. 